\title{
Deviation equation in Riemann-Cartan spacetime
}

\author{
Dirk Puetzfeld* \\ University of Bremen, Center of Applied Space Technology and Microgravity (ZARM), 28359 Bremen, Germany \\ Yuri N. Obukhovi \\ Theoretical Physics Laboratory, Nuclear Safety Institute, \\ Russian Academy of Sciences, B.Tulskaya 52, 115191 Moscow, Russia
}

(Dated: June 5, 2018)

\begin{abstract}
We derive a generalized deviation equation in Riemann-Cartan spacetime. The equation describes the dynamics of the connecting vector which links events on two general adjacent world lines. Our result is valid for any theory in a Riemann-Cartan background, in particular, it is applicable to a large class of gravitational theories which go beyond the general relativistic framework.
\end{abstract}

PACS numbers: 04.50.-h; 04.20.Cv; 04.25.-g

Keywords: Deviation equation; Approximation methods; Equations of motion; Riemann-Cartan spacetime

\section{INTRODUCTION}

Within the theory of General Relativity, the relative motion of test bodies is described by means of the geodesic deviation (Jacobi) equation [1] [4]. This equation only holds under certain assumptions and can be used only for the description of structureless neutral test bodies.

In a previous work [5], we have worked out generalized versions of the deviation equation, see also [6 28] for alternative derivations and generalizations. Our findings in [5] extended the range of applicability of the deviation equation to general world lines. However, the results were limited to theories in a Riemannian background. While such theories are justified in many physical situations, several modern gravitational theories [29 31] reach significantly beyond the Riemannian geometrical framework. In particular, it is already well known [32 34] that in the description of test bodies with intrinsic degrees of freedom - like spin - there is a natural coupling to the post-Riemannian features of spacetime. Therefore, in view of possible tests of gravitational theories by means of structured test bodies, a further extension of the deviation equation to post-Riemannian geometries seems to be overdue.

In this work we derive a generalized deviation equation in a Riemann-Cartan background, allowing for spacetimes endowed with torsion. This equation describes the dynamics of the connecting vector which links events on two general (adjacent) world lines. Our results are valid for any theory in a Riemann-Cartan background; in particular, they apply to Einstein-Cartan theory [35] as well as to Poincaré gauge theory 36.

The structure of the paper is as follows: In section [I] we briefly introduce the concepts needed in the derivation of the exact generalized deviation equation. This is followed by section III, in which we focus on the properties

* dirk.puetzfeld@zarm.uni-bremen.de http://puetzfeld.org

† obukhov@ibrae.ac.ru of a world function based on autoparallels in a RiemannCartan background. These results are then applied in section IV to arrive at an expanded approximate version of the deviation equation. In section $\mathrm{V}$ we discuss how different coordinate choices, depending on the underlying gravity theory, affect the interpretation and the operational value of the deviation equation. We conclude our paper in section VI with a discussion of the results obtained and with an outlook of their possible applications. Our notations and conventions are summarized in appendix $\mathrm{A}$ and table I Some details and intermediate results of our derivation are given in appendix B.

\section{WORLD FUNCTION AND DEVIATION EQUATION}

Let us briefly recapitulate the relevant steps that lead to the generalized deviation equation as derived in $[5]$. We want to compare two general curves $Y(t)$ and $X(\tilde{t})$ in an arbitrary spacetime manifold. Here $t$ and $\tilde{t}$ are general parameters, i.e. not necessarily the proper time on the given curves. In contrast to the Riemannian case, we now connect two points $x \in X$ and $y \in Y$ on the two curves by the autoparallel joining the two points (we assume that this autoparallel is unique). An autoparallel is a curve along which the velocity vector is transported parallel to itself with respect to the connection on the spacetime manifold. In a Riemannian space autoparallel curves coincide with geodesic lines.

Along the autoparallel we have the world function $\sigma$, and conceptually the closest object to the connecting vector between the two points is the covariant derivative of the world function, denoted at the point $y$ by $\sigma^{y}$, cf. fig. 1. Following our conventions, the reference curve will be $Y(t)$, and we define the generalized connecting vector to be

$$
\eta^{y}:=-\sigma^{y}
$$




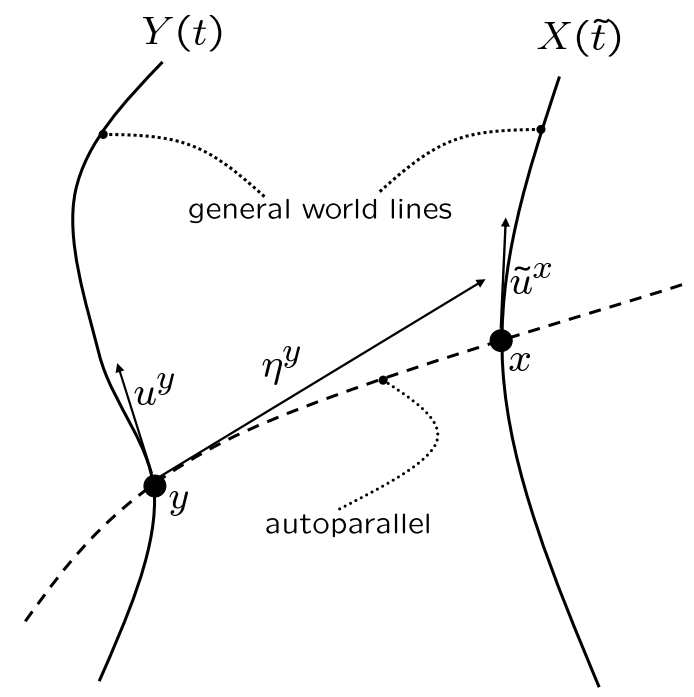

FIG. 1. Sketch of the two arbitrarily parametrized world lines $Y(t)$ and $X(\tilde{t})$ and the (dashed) autoparallel connecting two points on these world lines. The generalized deviation vector along the reference world line $Y$ is denoted by $\eta^{y}$.

Taking its covariant total derivative, we have

$$
\frac{D}{d t} \eta^{y_{1}}=-\sigma_{y_{2}}^{y_{1}} u^{y_{2}}-\sigma^{y_{1}}{ }_{x_{2}} \tilde{u}^{x_{2}} \frac{d \tilde{t}}{d t}
$$

where the velocities along the two curves $Y$ and $X$ are defined as $u^{y}:=d Y^{y} / d t$, and $\tilde{u}^{x}:=d X^{x} / d \tilde{t}$. Denoting the accelerations by $a^{y}:=D u^{y} / d t$, and $\tilde{a}^{x}:=D \tilde{u}^{x} / d \tilde{t}$, the second derivative becomes

$$
\begin{aligned}
& \frac{D^{2}}{d t^{2}} \eta^{y_{1}}=-\sigma_{y_{2} y_{3}}^{y_{1}} u^{y_{2}} u^{y_{3}}-2 \sigma^{y_{1}}{ }_{y_{2} x_{3}} u^{y_{2}} \tilde{u}^{x_{3}} \frac{d \tilde{t}}{d t} \\
& -\sigma_{y_{2}}^{y_{1}} a^{y_{2}}-\sigma_{x_{2} x_{3}}^{y_{1}} \tilde{u}^{x_{2}} \tilde{u}^{x_{3}}\left(\frac{d \tilde{t}}{d t}\right)^{2} \\
& -\sigma_{x_{2}}^{y_{1}} \tilde{a}^{x_{2}}\left(\frac{d \tilde{t}}{d t}\right)^{2}-\sigma_{x_{2}}^{y_{1}} \tilde{u}^{x_{2}} \frac{d^{2} \tilde{t}}{d t^{2}} .
\end{aligned}
$$

The generalized deviation equation is obtained from (3) by expressing all quantities therein along the reference word line $Y$. With the help of the inverse ${ }^{-1}$ - obtained from ${ }^{-1}{ }^{y_{1}}{ }_{x} \sigma_{y_{2}}^{x}=\delta^{y_{1}} y_{2}$ and ${ }^{-1} x_{1}{ }_{y} \sigma^{y} x_{2}=\delta^{x_{1}} x_{2}-$ and by defining the Jacobi propagators $H^{x_{1}} y_{2}:=-\sigma^{-1} x_{1} y_{2}$ and $K^{x_{1}} y_{2}:=-\sigma^{-1} x_{y_{1}} \sigma^{y_{1}}{ }_{y_{2}}$, the velocity along $X$ may be expressed as

$$
\tilde{u}^{x_{3}}=K_{y_{2}}^{x_{3}} u^{y_{2}} \frac{d t}{d \tilde{t}}-H_{y_{1}}^{x_{3}} \frac{D \sigma^{y_{1}}}{d t} \frac{d t}{d \tilde{t}}
$$

and inserted into (3)

$$
\begin{aligned}
& \frac{D^{2}}{d t^{2}} \eta^{y_{1}}=-\sigma_{y_{2} y_{3}}^{y_{1}} u^{y_{2}} u^{y_{3}}-\sigma_{y_{2}}^{y_{1}} a^{y_{2}}-\sigma_{x_{2}}^{y_{1}} \tilde{a}^{x_{2}}\left(\frac{d \tilde{t}}{d t}\right)^{2} \\
& -2 \sigma_{y_{2} x_{3}}^{y_{1}} u^{y_{2}}\left(K_{y_{4}}^{x_{3}} u^{y_{4}}-H_{y_{4}}^{x_{3}} \frac{D \sigma^{y_{4}}}{d t}\right) \\
& -\sigma^{y_{1}}{ }_{x_{2} x_{3}}\left(K_{y_{4}}^{x_{2}} u^{y_{4}}-H_{y_{4}}^{x_{2}} \frac{D \sigma^{y_{4}}}{d t}\right) \\
& \times\left(K_{y_{5}}^{x_{3}} u^{y_{5}}-H_{y_{5}}^{x_{3}} \frac{D \sigma^{y_{5}}}{d t}\right) \\
& -\sigma^{y_{1}} x_{2} \frac{d t}{d \tilde{t}} \frac{d^{2} \tilde{t}}{d t^{2}}\left(K_{y_{3}}^{x_{2}} u^{y_{3}}-H_{y_{3}}^{x_{2}} \frac{D \sigma^{y_{3}}}{d t}\right) .
\end{aligned}
$$

Note that we may determine the factor $d \tilde{t} / d t$ by requiring that the velocity along the curve $X$ is normalized, i.e. $\tilde{u}^{x} \tilde{u}_{x}=1$, in which case we have

$$
\frac{d \tilde{t}}{d t}=\tilde{u}_{x_{1}} K_{y_{2}}^{x_{1}} u^{y_{2}}-\tilde{u}_{x_{1}} H_{y_{2}}^{x_{1}} \frac{D \sigma^{y_{2}}}{d t} .
$$

Equation (5) is the exact generalized deviation equation, it is completely general and can be viewed as the extension of the standard geodesic deviation (Jacobi) equation to any order. In particular, it allows for a comparison of two general, i.e. not necessarily geodetic or autoparallel, world lines in spacetime.

\section{WORLD FUNCTION IN RIEMANN-CARTAN SPACETIME}

In this section we work out the basic properties of a world function $\sigma$ based on autoparallels in a RiemannCartan spacetime, which, in contrast to a Riemannian spacetime, is endowed with an asymmetric connection $\Gamma_{a b}{ }^{c}$. Relevant references that contain some results in a Riemann-Cartan context are [37 44].

For a world function $\sigma$ based on autoparallels, we have the following basic relations in the case of spacetimes with asymmetric connections:

$$
\begin{aligned}
\sigma^{x} \sigma_{x}=\sigma^{y} \sigma_{y} & =2 \sigma \\
\sigma^{x_{2}} \sigma_{x_{2}}{ }^{x_{1}} & =\sigma^{x_{1}}, \\
\sigma_{x_{1} x_{2}}-\sigma_{x_{2} x_{1}} & =T_{x_{1} x_{2}}^{x_{3}} \partial_{x_{3}} \sigma .
\end{aligned}
$$

Note, in particular, the change in (9) due to the presence of the spacetime torsion $T_{x_{1} x_{2}}{ }^{x_{3}}$, which leads to $\sigma_{x_{1} x_{2}} \neq$ $\sigma_{x_{2} x_{1}}$, in contrast to the symmetric Riemannian case, in which $\bar{\sigma}_{x_{1} x_{2}} \stackrel{\mathrm{s}}{=} \bar{\sigma}_{x_{2} x_{1}}$ holds 1

In many calculations the limiting behavior of a bitensor $B \ldots(x, y)$ as $x$ approaches the references point $y$ is

\footnotetext{
${ }^{1}$ We use "s" to indicate relations which only hold for symmetric connections and denote Riemannian objects by the overbar.
} 
required. This so-called coincidence limit of a bitensor $B \ldots(x, y)$ is a tensor

$$
[B \ldots]=\lim _{x \rightarrow y} B \ldots(x, y)
$$

at $y$ and will be denoted by square brackets. In particular, for a bitensor $B$ with arbitrary indices at different points (here just denoted by dots), we have the rule [45]

$$
\left[B_{\ldots,]_{j}}=\left[B_{\ldots ; y}\right]+\left[B_{\ldots ; x}\right] .\right.
$$

We collect the following useful identities for the world function $\sigma$ :

$$
\begin{aligned}
{[\sigma]=\left[\sigma_{x}\right]=\left[\sigma_{y}\right] } & =0, \\
{\left[\sigma_{x_{1} x_{2}}\right]=\left[\sigma_{y_{1} y_{2}}\right] } & =g_{y_{1} y_{2}}, \\
{\left[\sigma_{x_{1} y_{2}}\right]=\left[\sigma_{y_{1} x_{2}}\right] } & =-g_{y_{1} y_{2}}, \\
{\left[\sigma_{x_{3} x_{1} x_{2}}\right]+\left[\sigma_{x_{2} x_{1} x_{3}}\right] } & =0 .
\end{aligned}
$$

Note that up to the second covariant derivative the coincidence limits of the world function match those in spacetimes with symmetric connections. However, at the next (third) order the presence of the torsion leads to

$$
\left[\sigma_{x_{1} x_{2} x_{3}}\right]=\frac{1}{2}\left(T_{y_{1} y_{3} y_{2}}+T_{y_{2} y_{3} y_{1}}+T_{y_{1} y_{2} y_{3}}\right)=K_{y_{2} y_{1} y_{3}}
$$

where in the last line we made use of the contortion $K$, cf. also appendix $\mathrm{A}$ for an overview of the geometrical quantities With the help of (11), we obtain for the other combinations with three indices

$$
\begin{aligned}
& {\left[\sigma_{x_{1} x_{2} y_{3}}\right]=-\left[\sigma_{x_{1} x_{2} x_{3}}\right]=\left[\sigma_{y_{1} x_{2} y_{3}}\right]=\left[\sigma_{x_{2} x_{3} x_{1}}\right]=K_{y_{2} y_{3} y_{1}},} \\
& {\left[\sigma_{x_{1} y_{2} y_{3}}\right]=-\left[\sigma_{x_{2} x_{3} x_{1}}\right]=\left[\sigma_{x_{1} x_{3} x_{2}}\right]=\left[\sigma_{y_{1} x_{2} x_{3}}\right]=K_{y_{3} y_{1} y_{2}},} \\
& {\left[\sigma_{y_{1} y_{2} y_{3}}\right]=-\left[\sigma_{x_{3} x_{2} x_{1}}\right]=K_{y_{2} y_{1} y_{3}},} \\
& {\left[\sigma_{x_{1} y_{2} x_{3}}\right]=-\left[\sigma_{x_{1} x_{3} x_{2}}\right]=K_{y_{3} y_{2} y_{1}},} \\
& {\left[\sigma_{y_{1} y_{2} x_{3}}\right]=\left[\sigma_{x_{3} x_{2} x_{1}}\right]=K_{y_{2} y_{3} y_{1}} .}
\end{aligned}
$$

The non-vanishing of these limits leads to added complexity in subsequent calculations compared to the Riemannian case.

At the fourth order we have

$$
\begin{aligned}
& K_{y_{1}}{ }^{y}{ }_{y_{2}} K_{y_{3} y_{4}}+K_{y_{1}}{ }^{y}{ }_{y_{3}} K_{y_{2} y_{y_{4}}}+K_{y_{1}}{ }^{y}{ }_{y_{4}} K_{y_{2} y y_{4}} \\
& +\left[\sigma_{x_{4} x_{1} x_{2} x_{3}}\right]+\left[\sigma_{x_{3} x_{1} x_{2} x_{4}}\right]+\left[\sigma_{x_{2} x_{1} x_{3} x_{4}}\right]=0,
\end{aligned}
$$

and in particular

$$
\begin{aligned}
{\left[\sigma_{x_{1} x_{2} x_{3} x_{4}}\right]=} & \frac{1}{3} \nabla_{y_{1}}\left(K_{y_{3} y_{2} y_{4}}+K_{y_{4} y_{2} y_{3}}\right)+\frac{1}{3} \nabla_{y_{3}}\left(3 K_{y_{2} y_{1} y_{4}}-K_{y_{1} y_{2} y_{4}}\right)+\frac{1}{3} \nabla_{y_{4}}\left(3 K_{y_{2} y_{1} y_{3}}-K_{y_{1} y_{2} y_{3}}\right)+\pi_{y_{1} y_{2} y_{3} y_{4}} \\
{\left[\sigma_{x_{1} x_{2} x_{3} y_{4}}\right]=} & -\frac{1}{3} \nabla_{y_{1}}\left(K_{y_{3} y_{2} y_{4}}+K_{y_{4} y_{2} y_{3}}\right)-\frac{1}{3} \nabla_{y_{3}}\left(3 K_{y_{2} y_{1} y_{4}}-K_{y_{1} y_{2} y_{4}}\right)+\frac{1}{3} \nabla_{y_{4}} K_{y_{1} y_{2} y_{3}}-\pi_{y_{1} y_{2} y_{3} y_{4}} \\
{\left[\sigma_{x_{1} x_{2} y_{3} y_{4}}\right]=} & \frac{1}{3} \nabla_{y_{1}}\left(K_{y_{4} y_{2} y_{3}}+K_{y_{3} y_{2} y_{4}}\right)-\frac{1}{3} \nabla_{y_{4}} K_{y_{1} y_{2} y_{3}}-\frac{1}{3} \nabla_{y_{3}} K_{y_{1} y_{2} y_{4}}+\pi_{y_{1} y_{2} y_{4} y_{3}} \\
{\left[\sigma_{x_{1} y_{2} y_{3} y_{4}}\right]=} & -\frac{1}{3} \nabla_{y_{1}}\left(K_{y_{3} y_{4} y_{2}}+K_{y_{2} y_{4} y_{3}}\right)+\frac{1}{3} \nabla_{y_{3}} K_{y_{1} y_{4} y_{2}}+\frac{1}{3} \nabla_{y_{2}} K_{y_{1} y_{4} y_{3}}+\nabla_{y_{4}} K_{y_{3} y_{1} y_{2}}-\pi_{y_{1} y_{4} y_{3} y_{2}} \\
{\left[\sigma_{y_{1} y_{2} y_{3} y_{4}}\right]=} & \frac{1}{3} \nabla_{y_{4}}\left(-2 K_{y_{2} y_{3} y_{1}}+K_{y_{1} y_{3} y_{2}}\right)-\frac{1}{3} \nabla_{y_{2}} K_{y_{4} y_{3} y_{1}}-\frac{1}{3} \nabla_{y_{1}} K_{y_{4} y_{3} y_{2}}-\nabla_{y_{3}} K_{y_{2} y_{4} y_{1}}+\pi_{y_{4} y_{3} y_{2} y_{1}} \\
\pi_{y_{1} y_{2} y_{3} y_{4}}:= & \frac{1}{3}\left[K_{y_{1} y_{2}}{ }^{y}\left(K_{y_{3} y_{4} y}+K_{y_{4} y_{3} y}\right)-K_{y_{1} y_{3}}{ }^{y}\left(K_{y_{4} y_{2} y}+K_{y_{2} y_{4}}\right)-K_{y_{1} y_{4}}{ }^{y}\left(K_{y_{3} y_{2} y}+K_{y y_{2} y_{3}}\right)\right. \\
& \left.-3 K_{y_{2} y_{1}}{ }^{y} K_{y_{3} y_{4} y}+K_{y_{3} y_{1}}{ }^{y} K_{y y_{2} y_{4}}+K_{y_{4} y_{1}}{ }^{y} K_{y_{2} y_{3}}+R_{y_{1} y_{3} y_{2} y_{4}}+R_{y_{1} y_{4} y_{2} y_{3}}\right]
\end{aligned}
$$

Again, we note the added complexity compared to the Riemannian case, in which we have $\left[\sigma_{x_{1} x_{2} x_{3} x_{4}}\right] \stackrel{\mathrm{s}}{=}$ $\frac{1}{3}\left(\bar{R}_{y_{2} y_{4} y_{1} y_{3}}+\bar{R}_{y_{3} y_{2} y_{1} y_{4}}\right)$ at the fourth order. In particular, we observe the occurrence of derivatives of the contortion in (19)-(23).

Finally, let us collect the basic properties of the socalled parallel propagator $g^{y} x:=e_{(a)}^{y} e_{x}^{(a)}$, defined in

\footnotetext{
2 The contortion $K_{y_{2} y_{1} y_{3}}$ should not be confused with the Jacobi propagator $K^{x}$.
}

terms of a parallely propagated tetrad $e_{(a)}^{y}$, which in turn allows for the transport of objects, i.e. $V^{y}=$ $g^{y}{ }_{x} V^{x}, \quad V^{y_{1} y_{2}}=g^{y_{1}}{ }_{x_{1}} g^{y_{2}}{ }_{x_{2}} V^{x_{1} x_{2}}$, etc., along an autoparallel:

$$
\begin{aligned}
g^{y_{1}}{ }_{x} g^{x}{ }_{y_{2}} & =\delta^{y_{1}}{ }_{{ }_{2}}, \quad g^{x_{1}} g_{y}^{y}{ }_{x_{2}}=\delta^{x_{1}}{ }_{x_{2}}, \\
\sigma^{x} \nabla_{x} g^{x_{1}}{ }_{y_{1}} & =\sigma^{y} \nabla_{y} g^{x_{1}}{ }_{y_{1}}=0, \\
\sigma^{x} \nabla_{x} g^{y_{1}}{ }_{x_{1}} & =\sigma^{y} \nabla_{y} g^{y_{1}}{ }_{x_{1}}=0, \\
\sigma_{x} & =-g^{y}{ }_{x} \sigma_{y}, \quad \sigma_{y}=-g^{x}{ }_{y} \sigma_{x} .
\end{aligned}
$$

Note, in particular, the coincidence limits of its deriva- 
tives

$$
\begin{aligned}
& {\left[g^{x_{0}}{ }_{y_{1}}\right]=\delta^{y_{0}}{ }_{y_{1}},} \\
& {\left[g^{x_{0}}{ }_{y_{1} ; x_{2}}\right]=\left[g^{x_{0}} y_{1} ; y_{2}\right]=0,} \\
& {\left[g^{x_{0}} y_{1} ; x_{2} x_{3}\right]=-\left[g^{x_{0}}{ }_{y_{1} ; x_{2} y_{3}}\right]=\left[g^{x_{0}}{ }_{y_{1} ; x_{2} x_{3}}\right]} \\
& =-\left[g_{y_{1} ; y_{2} y_{3}}^{x_{0}}\right]=\frac{1}{2} R^{y_{0}}{ }_{y_{1} y_{2} y_{3}} .
\end{aligned}
$$

In the next section we will derive an expanded approximate version of the deviation equation. For this we first work out the expanded version of quantities around the reference world line $Y$. In particular, we make use of the covariant expansion technique [45, 46] on the basis of the autoparallel world function.

\section{EXPANDED RIEMANN-CARTAN DEVIATION EQUATION}

For a general bitensor $B$... with a given index structure, we have the following general expansion, up to the third order (in powers of $\sigma^{y}$ ):

$$
\begin{aligned}
B_{y_{1} \ldots y_{n}}= & A_{y_{1} \ldots y_{n}}+A_{y_{1} \ldots y_{n+1}} \sigma^{y_{n+1}} \\
& +\frac{1}{2} A_{y_{1} \ldots y_{n+1} y_{n+2}} \sigma^{y_{n+1}} \sigma^{y_{n+2}}+\mathcal{O}\left(\sigma^{3}\right), \\
A_{y_{1} \ldots y_{n}}:= & {\left[B_{y_{1} \ldots y_{n}}\right] } \\
A_{y_{1} \ldots y_{n+1}}:= & {\left[B_{y_{1} \ldots y_{n} ; y_{n+1}}\right]-A_{y_{1} \ldots y_{n} ; y_{n+1}}, } \\
A_{y_{1} \ldots y_{n+2}}:= & {\left[B_{y_{1} \ldots y_{n} ; y_{n+1} y_{n+2}}\right]-A_{y_{1} \ldots y_{n} y_{0}}\left[\sigma^{y_{0}} y_{n+1} y_{n+2}\right] } \\
& -A_{y_{1} \ldots y_{n} ; y_{n+1} y_{n+2}}-2 A_{y_{1} \ldots y_{n}\left(y_{n+1} ; y_{n+2}\right)} .(34)
\end{aligned}
$$

With the help of (31) we are able to iteratively expand any bitensor to any order, provided the coincidence limits entering the expansion coefficients can be calculated. The expansion for bitensors with mixed index structure can be obtained from transporting the indices in (31) by means of the parallel propagator.

To develop an approximate form of the generalized deviation equation (5) up to the second order, we need the following expansions - note that we give some explicit intermediate results in appendix B - of the derivatives of the world function:

$$
\begin{aligned}
\sigma_{y_{1} y_{2}} & =g_{y_{1} y_{2}}+K_{y_{2} y_{1} y_{3}} \sigma^{y_{3}}+\mathcal{O}\left(\sigma^{2}\right) \\
\sigma_{y_{1} x_{2}} & =-g_{y_{1} x_{2}}+g_{x_{2}}{ }^{y} K_{y_{3} y y_{1}} \sigma^{y_{3}}+\mathcal{O}\left(\sigma^{2}\right) \\
\sigma_{y_{1} y_{2} y_{3}} & =K_{y_{2} y_{1} y_{3}}+\frac{1}{3}\left[\nabla_{y_{4}}\left(K_{y_{2} y_{3} y_{1}}+K_{y_{1} y_{3} y_{2}}\right)\right. \\
& -\nabla_{y_{2}} K_{y_{4} y_{3} y_{1}}-\nabla_{y_{1}} K_{y_{4} y_{3} y_{2}}-3 \nabla_{y_{3}} K_{y_{2} y_{4} y_{1}} \\
& \left.+3 \pi_{y_{4} y_{3} y_{2} y_{1}}\right] \sigma^{y_{4}}+\mathcal{O}\left(\sigma^{2}\right)
\end{aligned}
$$

$$
\begin{aligned}
\sigma_{y_{1} y_{2} x_{3}} & =g_{x_{3}}{ }^{y_{3}}\left\{K_{y_{2} y_{3} y_{1}}-\frac{1}{3}\left[\nabla_{y_{3}}\left(K_{y_{2} y_{4} y_{1}}+K_{y_{1} y_{4} y_{2}}\right)\right.\right. \\
& -\nabla_{y_{2}} K_{y_{3} y_{4} y_{1}}-\nabla_{y_{1}} K_{y_{3} y_{4} y_{2}} \\
& \left.\left.+3 \pi_{y_{3} y_{4} y_{2} y_{1}}\right] \sigma^{y_{4}}\right\}+\mathcal{O}\left(\sigma^{2}\right) \\
\sigma_{y_{1} x_{2} x_{3}} & =g_{x_{2}}{ }_{y_{2}} g_{x_{3}}{ }^{y_{3}}\left\{K_{y_{3} y_{1} y_{2}}\right. \\
& +\frac{1}{3}\left[\nabla_{y_{2}}\left(K_{y_{4} y_{3} y_{1}}+K_{y_{1} y_{3} y_{4}}\right)\right. \\
& -\nabla_{y_{4}}\left(K_{y_{2} y_{3} y_{1}}+3 K_{y_{3} y_{1} y_{2}}\right) \\
& \left.\left.-\nabla_{y_{1}} K_{y_{2} y_{3} y_{4}}+3 \pi_{y_{2} y_{3} y_{4} y_{1}}\right] \sigma^{y_{4}}\right\}+\mathcal{O}\left(\sigma^{2}\right) \cdot(39
\end{aligned}
$$

The Jacobi propagators are approximated as

$$
\begin{aligned}
& H_{y_{2}}^{x_{1}}=g_{y_{2}}^{x_{1}}+K_{y_{3} y_{2}}^{x_{1}} \sigma^{y_{3}}+\mathcal{O}\left(\sigma^{2}\right), \\
& K_{y_{2}}^{x_{1}}=g^{x_{1}}{ }_{y_{2}}+\left(K_{y_{2}}{ }^{x_{1}}{ }_{y_{3}}+K_{y_{3} y_{2}}^{x_{1}}\right) \sigma^{y_{3}}+\mathcal{O}\left(\sigma^{2}\right) \text {, }
\end{aligned}
$$

which in turn allows for an expansion of the recurring term entering (5):

$$
\begin{aligned}
& \left(K_{y_{2}}^{x_{1}} u^{y_{2}}-H_{y_{2}}^{x_{1}} \frac{D \sigma^{y_{2}}}{d t}\right)=g_{y^{\prime}}^{x_{1}}\left[u^{y^{\prime}}-\frac{D \sigma^{y^{\prime}}}{d t}\right. \\
& \left.+\left(K_{y_{2}}{ }^{y^{\prime}}{ }_{y_{3}}+K_{y_{3} y_{2}} y^{\prime}\right) u^{y_{2}} \sigma^{y_{3}}\right]+\mathcal{O}\left(\sigma^{2}\right) .
\end{aligned}
$$

\section{A. Synchronous parametrization}

Before writing down the expanded version of the generalized deviation equation, we will simplify the latter by choosing a proper parametrization of the neighboring curves. The factors with the derivatives of the parameters $t$ and $\tilde{t}$ appear in (5) due to the non-synchronous parametrization of the two curves. It is possible to make things simpler by introducing the synchronization of parametrization. Namely, we start by rewriting the velocity as

$$
u^{y}=\frac{d Y^{y}}{d t}=\frac{d \tilde{t}}{d t} \frac{d Y^{y}}{d \tilde{t}} .
$$

That is, we now parametrize the position on the first curve by the same variable $\tilde{t}$ that is used on the second curve. Accordingly, we denote

$$
\underline{\sim}^{y}=\frac{d Y^{y}}{d \tilde{t}} .
$$

By differentiation, we then derive

$$
a^{y}=\frac{d^{2} \tilde{t}}{d t^{2}} \stackrel{u}{y}^{y}+\left(\frac{d \tilde{t}}{d t}\right)^{2} \stackrel{a}{a}^{y}
$$


where

$$
\stackrel{\sim}{a}^{y}=\frac{D}{d \tilde{t}} u^{y}=\frac{D^{2} Y^{y}}{d \tilde{t}^{2}}
$$

Analogously, we derive for the derivative of the deviation vector

$$
\frac{D^{2} \eta^{y}}{d t^{2}}=\frac{d^{2} \tilde{t}}{d t^{2}} \frac{D \eta^{y}}{d \tilde{t}}+\left(\frac{d \tilde{t}}{d t}\right)^{2} \frac{D^{2} \eta^{y}}{d \tilde{t}^{2}}
$$

Now everything is synchronous in the sense that both curves are parametrized by $\tilde{t}$.

As a result, the exact deviation equation (5) is recast into a simpler form

$$
\begin{aligned}
& \frac{D^{2}}{d \tilde{t}^{2}} \eta^{y_{1}}=-\sigma_{y_{2}}^{y_{1}} a^{y_{2}}-\sigma^{y_{1}}{ }_{x_{2}} \tilde{a}^{x_{2}}-\sigma^{y_{1}}{ }_{y_{2}} y_{3} u_{\sim}^{y_{2}} \underline{u}^{y_{3}} \\
& -2 \sigma^{y_{1}}{ }_{y_{2} x_{3}}{\underset{\sim}{u}}^{y_{2}}\left(K_{y_{4}}^{x_{3}}{\underset{\sim}{y_{4}}}^{y_{4}}-H_{y_{4}}^{x_{3}} \frac{D \sigma^{y_{4}}}{d \tilde{t}}\right) \\
& -\sigma^{y_{1}}{ }_{x_{2} x_{3}}\left(K^{x_{2}}{ }_{y_{4}} u^{y_{4}}-H_{y_{4}}^{x_{2}} \frac{D \sigma^{y_{4}}}{d \tilde{t}}\right)
\end{aligned}
$$

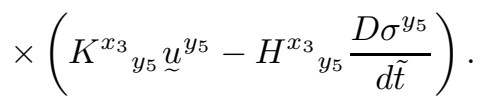

\section{B. Explicit expansion of the deviation equation}

Substituting the expansions (35)-(42) into (48), we obtain the final result

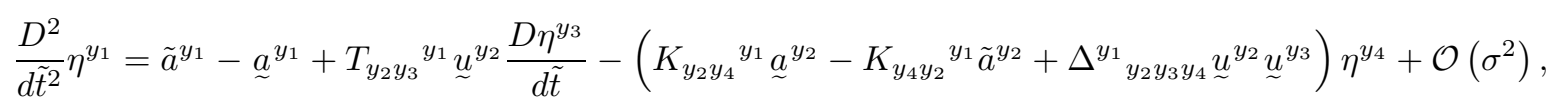

where we introduced the abbreviation

$$
\Delta_{y_{1} y_{2} y_{3} y_{4}}:=2 \pi_{y_{3} y_{4} y_{2} y_{1}}-\pi_{y_{4} y_{3} y_{2} y_{1}}-\pi_{y_{2} y_{3} y_{4} y_{1}}+T_{y^{\prime} y_{2} y_{1}} T_{y_{4} y_{3}}{ }^{\prime}-2 \nabla_{y_{2}} K_{\left(y_{1} y_{3}\right) y_{4}}+\nabla_{y_{1}} K_{y_{2} y_{3} y_{4}}-\nabla_{y_{4}} K_{y_{2} y_{3} y_{1}} .
$$

It should be understood that the last expression is contracted with ${\underset{\sim}{u}}^{y_{2}}{\underset{\sim}{u}}^{y_{3}}$ and hence the symmetrization is naturally imposed on the indices $\left(y_{2} y_{3}\right)$.

Equation (49) allows for the comparison of two general world lines in Riemann-Cartan spacetime, which are not necessarily geodetic or autoparallel. It therefore represents the generalization of the deviation equation derived in $[5,(35)]$.

\section{Riemannian case}

A great simplification is achieved in a Riemannian background, when

$$
\begin{aligned}
\bar{\Delta}_{y_{1} y_{2} y_{3} y_{4}} & =2 \bar{\pi}_{y_{3} y_{4} y_{2} y_{1}}-\bar{\pi}_{y_{4} y_{3} y_{2} y_{1}}-\bar{\pi}_{y_{2} y_{3} y_{4} y_{1}} \\
& =\bar{R}_{y_{1} y_{3} y_{2} y_{4}},
\end{aligned}
$$

and (49) is reduced to

$$
\frac{D^{2}}{d \tilde{t}^{2}} \eta^{y_{1}} \stackrel{\mathrm{s}}{=} \tilde{a}^{y_{1}}-{\underset{\sim}{a}}^{y_{1}}-\bar{R}_{y_{2} y_{3} y_{4}}^{y_{1}}{\stackrel{\sim}{u_{2}}}_{\underbrace{u_{3}}}^{y_{3}} \eta^{y_{4}}+\mathcal{O}\left(\sigma^{2}\right) .
$$

Along geodesic curves, this equation is further reduced to the well known geodesic deviation (Jacobi) equation.

\section{CHOICE OF COORDINATES}

To utilize the deviation equation for measurements or in a gravitational compass setup [5, 45, 47, 48], the oc- curring covariant total derivatives need to be rewritten and an appropriate coordinate choice needs to be made. The lhs of the deviation equation takes the form:

$$
\begin{aligned}
\frac{D^{2} \eta_{a}}{d t^{2}}= & \dot{u}^{b} \nabla_{b} \eta_{a}+\stackrel{\circ}{\eta}_{a}-2 u^{b} \Gamma_{b a}{ }^{d} \stackrel{\circ}{\eta}_{d}-u^{b} u^{c} \Gamma_{c b}{ }^{d} \partial_{d} \eta_{a} \\
& -u^{b} u^{c} \eta_{e}\left(\partial_{c} \Gamma_{b a}{ }^{e}-\Gamma_{c b}{ }^{d} \Gamma_{d a}{ }^{e}-\Gamma_{c a}{ }^{d} \Gamma_{b d}{ }^{e}\right)
\end{aligned}
$$

Here we used $\stackrel{\circ}{\eta}^{a}:=d \eta^{a} / d t$ for the standard total derivative.

Observe that the first term on the rhs vanishes in the case of autoparallel curves $\left(\dot{u}^{a}:=D u^{a} / d t=0\right)$. Also note the symmetrization of the connection imposed by the velocities in some terms.

Rewriting the connection in terms of the contortion and switching to normal coordinates [49 [55] along the world line, which we assume to be an autoparallel, yields

$$
\begin{aligned}
& \frac{D^{2} \eta_{a}}{d t^{2}} \stackrel{\left.\right|_{Y}}{=} \stackrel{\circ}{\eta}_{a}+2 u^{b} K_{b a}{ }^{d} \stackrel{\circ}{\eta}_{d}+u^{b} u^{c} K_{c b}{ }^{d} \partial_{d} \eta_{a} \\
& +u^{b} u^{c} \eta_{e}\left(\partial_{c} K_{b a}{ }^{e}-\frac{2}{3} \bar{R}_{c(b a)}{ }^{e}+K_{c b}{ }^{d} K_{d a}{ }^{e}-K_{c a}{ }^{d} K_{b d}{ }^{e}\right) .
\end{aligned}
$$

Note the appearance of a term containing the partial (not ordinary total) derivative of the deviation vector, in contrast to the Riemannian case.

The first term in the second line may be rewritten as an ordinary total derivative, i.e. $u^{b} u^{c} \eta_{e} \partial_{c} K_{b a}{ }^{e}=u^{b} \eta_{e} \stackrel{\circ}{K} b a{ }^{e}$, 
but this is still inconvenient when recalling the compass equation, which will contain terms with covariant derivatives of the contortion.

\section{A. Operational interpretation}

At this point, some thoughts about the operational interpretation of the coordinate choice are in order. In particular, it should be stressed that so far we did not specify any physical theory in which the deviation equation (48) should be applied. Stated the other way round, the derived deviation equation is of completely geometrical nature, i.e. it describes the change of the deviation vector between points on two general curves in RiemannCartan spacetime.

From the mathematical perspective, the choice of coordinates should be solely guided by the simplicity of the resulting equation. In this sense, our previous choice of normal coordinates appears to be appropriate. But what about the physical interpretation or, better, the operational realization of such coordinates?

Let us recall the coordinate choice in General Relativity in a Riemannian background. In this case, normal coordinates also have a clear operational meaning, which is related to the motion of structureless test bodies in General Relativity. As is well known, such test bodies move along the geodesic equation. In other words, we could - at least in principle - identify a normal coordinate system by the local observation of test bodies. If other external forces are absent, normal coordinates will locally ${ }^{3}$ lead to straight line motion of test bodies. In this sense, there is a clear operational procedure for the realization of normal coordinates.

However, now we are in a more general situation, since we have not yet specified which gravitational theory we are considering in the geometrical Riemann-Cartan background. The physical choice of a gravity theory will be crucial for the operational realization of the coordinates. Recall the form of the equations of motion for a very large class [33, 34] of gravitational theories, which also allow for additional internal degrees of freedom, in particular for spin. In this case, the equations of motion are no longer given by the geodesic equation or, as it is sometimes erroneously postulated in the literature, by the autoparallel equation. In such theories, test bodies exhibit an additional spin-curvature coupling, which leads to non-geodesic motion, even locally.

How does this impact the operational realization of normal coordinates in such theories? Mainly, one just has to be aware of the fact that for the experimental realization of the normal coordinates, one now has to make sure to use the correct equation of motion and,

${ }^{3}$ Here, "locally" refers to the observers laboratory on the reference world line. consequently, the correct type of test body. Taking the example of a theory with spin-curvature coupling, like Einstein-Cartan theory, this would eventually lead to the usage of test bodies with vanishing spin - since those still move on standard geodesics, and therefore lead to an identical procedure as in the general relativistic case, i.e. one adopts coordinates in which the motion of those test bodies becomes rectilinear.

\section{CONCLUSIONS \& OUTLOOK}

In this work we investigated the generalization of the deviation equation in a Riemann-Cartan geometry. As a novel technical result, we have developed Synge's world function approach in the non-Riemannian spacetime with curvature and torsion. Our expanded version of the deviation equation (48) can be directly compared to result in the Riemannian context [5]. The generalization should serve as a foundation for the test of gravitational theories that make use of post-Riemannian geometrical structures.

As we have discussed in detail, the operational usability of the Riemann-Cartan deviation equation differs from the one in a general relativistic context, which was also noticed quite early in 29]. In particular, it remains to be shown which additional concepts and assumptions are needed in order to fully realize a gravitational compass [5, 45, 47, 48] in a Riemann-Cartan background. In contrast to the Riemannian case, an algebraic realization of a gravitational compass on the basis of the deviation equation is out of the question due to the appearance of derivatives of the torsion even at the lowest orders.

An interesting question for future works is the possible application of (48) to the analysis of motion of (micro)structured test bodies. In particular, it seems worthwhile to search for new ways to map the gravitational field with the help of such a deviation equation and work out its implications for various applications, aiming for novel tests of relativistic gravity theories.

\section{ACKNOWLEDGEMENTS}

This work was supported by the Deutsche Forschungsgemeinschaft (DFG) through the grant PU 461/1-1 (D.P.). The work of Y.N.O. was partially supported by PIER ("Partnership for Innovation, Education and Research" between DESY and Universität Hamburg) and by the Russian Foundation for Basic Research (Grant No. 16-02-00844-A). 
TABLE I. Directory of symbols.

\begin{tabular}{|c|c|}
\hline Symbol & Explanation \\
\hline \multicolumn{2}{|c|}{ Geometrical quantities } \\
\hline$g_{a b}$ & Metric \\
\hline$\delta_{b}^{a}$ & Kronecker symbol \\
\hline$x^{a}, y^{a}$ & Coordinates \\
\hline$\Gamma_{a b}^{c}$ & Connection \\
\hline $\bar{\Gamma}_{a b}^{c}$ & Levi-Civita connection \\
\hline$R_{a b c}{ }^{d}$ & Curvature \\
\hline$T_{a b}{ }^{c}$ & Torsion \\
\hline$K_{a b}^{c}$ & Contortion \\
\hline$\sigma$ & World function \\
\hline$\eta^{y}$ & Deviation vector \\
\hline$g^{y_{0}}{ }_{x_{0}}$ & Parallel propagator \\
\hline \multicolumn{2}{|l|}{ Misc } \\
\hline$Y(t), X(\tilde{t})$ & (Reference) world line \\
\hline$u^{a}$ & Velocity \\
\hline$a^{b}$ & Acceleration \\
\hline$K^{x}{ }_{y}, H_{y}^{x}$ & Jacobi propagators \\
\hline$A_{y_{1} \ldots y_{n}}$ & Expansion coefficient \\
\hline$\pi_{y_{1} y_{2} y_{3} y_{4}}$ & Auxiliary quantities \\
\hline \multicolumn{2}{|l|}{ Operators } \\
\hline$\partial_{i}, “, "$ & Partial derivative \\
\hline$\nabla_{i}$, " ;" & Covariant derivative \\
\hline$\frac{D}{d t}=" \cdots "$ & Total cov. derivative \\
\hline$\frac{d}{d t}="{ }^{\circ} "$ & Total derivative \\
\hline$"[\ldots] "$ & Coincidence limit \\
\hline “", & Riemannian object \\
\hline
\end{tabular}

\section{Appendix A: Notation \& Conventions}

The curvature and the torsion are defined w.r.t. the general connection $\Gamma_{a b}{ }^{c}$ as follows:

$$
\begin{aligned}
R_{a b c}{ }^{d} & :=\partial_{a} \Gamma_{b c}{ }^{d}-\partial_{b} \Gamma_{a c}{ }^{d}+\Gamma_{a n}{ }^{d} \Gamma_{b c}{ }^{n}-\Gamma_{b n}{ }^{d} \Gamma_{a c}{ }^{n}, \\
T_{a b}{ }^{c} & :=\Gamma_{a b}{ }^{c}-\Gamma_{b a}{ }^{c} .
\end{aligned}
$$

The symmetric Levi-Civita connection $\bar{\Gamma}_{k j}{ }^{i}$, as well as all other Riemannian quantities, are denoted by an additional overline. For a general tensor $A$ of $\operatorname{rank}(n, l)$ the commutator of the covariant derivative thus takes the form:

$$
\begin{gathered}
\left(\nabla_{a} \nabla_{b}-\nabla_{b} \nabla_{a}\right) A^{c_{1} \ldots c_{n}}{ }_{d_{1} \ldots d_{l}}=-T_{a b}{ }^{e} \nabla_{e} A^{c_{1} \ldots c_{k}} d_{1} \ldots d_{l} \\
+\sum_{i=1}^{k} R_{a b e}{ }^{c_{i}} A^{c_{1} \ldots e \ldots c_{k}} d_{1} \ldots d_{l}-\sum_{j=1}^{l} R_{a b d_{j}}{ }^{e} A^{c_{1} \ldots c_{k}}{ }_{d_{1} \ldots e \ldots d_{l}} .
\end{gathered}
$$

In addition to the torsion, we define the contortion $K_{k j}{ }^{i}$ with the following properties

$$
\begin{aligned}
K_{k j}{ }^{i} & :=\bar{\Gamma}_{k j}{ }^{i}-\Gamma_{k j}{ }^{i}, \\
K_{k j i} & =-\frac{1}{2}\left(T_{k j i}+T_{i k j}+T_{i j k}\right), \\
T_{k j}{ }^{i} & =-2 K_{[k j]}^{i} .
\end{aligned}
$$

The signature of the spacetime metric is assumed to be $(+1,-1,-1,-1)$.

As usual, $\sigma^{y} x_{1} \ldots y_{2} \ldots:=\nabla_{x_{1}} \ldots \nabla_{y_{2}} \ldots\left(\sigma^{y}\right)$ denote the higher-order covariant derivatives of the world function.

\section{Appendix B: Intermediate results}

Here we give some intermediate results of the derivation of the expansions the world function derivatives around the reference world line $Y$ :

$$
\begin{aligned}
\sigma_{y_{1} y_{2}}= & g_{y_{1} y_{2}}+\left[\sigma_{y_{1} y_{2} y_{3}}\right] \sigma^{y_{3}}+\left(\frac{1}{2}\left[\sigma_{y_{1} y_{2} y_{3} y_{4}}\right]-\left[\sigma_{y_{1} y_{2} y_{3}}\right]_{; y_{4}}-\frac{1}{2}\left[\sigma_{y_{1} y_{2} y_{5}}\right]\left[\sigma_{y_{3} y_{4}}^{y_{5}}\right]\right) \sigma^{y_{3}} \sigma^{y_{4}}+\mathcal{O}\left(\sigma^{3}\right), \\
\sigma_{y_{1} y_{2} y_{3}}= & {\left[\sigma_{y_{1} y_{2} y_{3}}\right]+\left(\left[\sigma_{y_{1} y_{2} y_{3} y_{4}}\right]-\left[\sigma_{y_{1} y_{2} y_{3}}\right]_{; y_{4}}\right) \sigma^{y_{4}}+\frac{1}{2}\left(\left[\sigma_{y_{1} y_{2} y_{3} y_{4} y_{5}}\right]-\left[\sigma_{y_{1} y_{2} y_{3}}\right]_{y_{4} y_{4}}-2\left[\sigma_{y_{1} y_{2} y_{3} y_{4}}\right]_{; y_{5}}\right.} \\
& \left.-\left[\sigma_{\left.y_{1} y_{2} y_{3} y_{0}\right]}\right]\left[\sigma^{y_{0}}{ }_{y_{4} y_{5}}\right]+\left[\sigma_{y_{1} y_{2} y_{3}}\right]_{y_{0}}\left[\sigma^{y_{0}}{ }_{y_{4} y_{5}}\right]\right) \sigma^{y_{4}} \sigma^{y_{5}}+\mathcal{O}\left(\sigma^{3}\right), \\
\sigma_{y_{1} x_{2}}= & -g_{y_{1} x_{2}}+g_{x_{2}}{ }^{y}\left[\sigma_{y_{1} x y_{3}}\right] \sigma^{y_{3}}+\frac{1}{2}\left(g_{x_{2}}{ }^{y}\left[\sigma_{y_{1} x y_{3} y_{4}}\right]-g_{x_{2}}{ }^{y_{2}} g_{y_{1} y}\left[g_{y_{2}}{ }^{x} ; y_{3} y_{4}\right]\right. \\
& \left.-2 g_{x_{2}}{ }^{y}\left[\sigma_{y_{1} x\left(y_{3}\right.}\right]_{\left.; y_{4}\right)}-g_{x_{2}}{ }^{y}\left[\sigma_{y_{1} x y_{5}}\right]\left[\sigma^{y_{5}}{ }_{y_{3} y_{4}}\right]\right) \sigma^{y_{3}} \sigma^{y_{4}}+\mathcal{O}\left(\sigma^{3}\right) .
\end{aligned}
$$


[1] T. Levi-Civita. Sur l'écart géodésique. Math. Ann., 97: 291, 1927.

[2] J. L. Synge. The first and second variations of the length integral in Riemannian space. Proc. Lond. Math. Soc., 25:247, 1926.

[3] J. L. Synge. On the geometry of dynamics. Phil. Trans. R. Soc. Lond. A, 226:31, 1927.

[4] F. A. E. Pirani. On the physical significance of the Riemann tensor. Acta Phys. Pol., 15:389, 1956.

[5] D. Puetzfeld and Y. N. Obukhov. Generalized deviation equation and determination of the curvature in General Relativity. Phys. Rev. D, 93:044073, 2016.

[6] J. Plebański. Conformal geodesic deviations. Acta Phys. Pol., 28:141, 1965.

[7] D. E. Hodgkinson. A modified theory of geodesic deviation. Gen. Rel. Grav., 3:351, 1972.

[8] S. L. Bażański. The relative energy of test particles in General Relativity. Nova Acta Leopoldina, 39:215, 1974.

[9] S. L. Bażański. A geometric formulation of the Taylor theorem for curves on affine manifolds. J. Math. Phys. (N. Y.), 17:217, 1976.

[10] S. L. Bażański. Kinematics of relative motion of test particles in general relativity. Ann. H. Poin. A, 27:115, 1977.

[11] S. L. Bażański. Dynamics of relative motion of test particles in general relativity. Ann. H. Poin. A, 27:145, 1977.

[12] M. Novello, I. Damião Soares, and J. M. Salim. On Jacobi fields. Gen. Rel. Grav, 8:95, 1977.

[13] A. N. Aleksandrov and K. A. Piragas. Geodesic structure: I. Relative dynamics of geodesics. Theoretical and Mathematical Physics, 38:48, 1978.

[14] S. Manoff. Lie derivatives and deviation equations in Riemannian spaces. Gen. Rel. Grav, 11:189, 1979.

[15] R. Schattner and M. Trümper. World vectors, Jacobi vectors and Jacobi one-forms on a manifold with a linear symmetric connection. J. Phys. A: Math. Gen., 14:2345, 1981.

[16] N. S. Swaminarayan and J. L. Safko. A coordinate-free derivation of a generalized geodesic deviation equation. J. Math. Phys. (N. Y.), 24:883, 1983.

[17] B. Schutz. On generalized equations of geodesic deviation. In: Galaxies, Axisymmetric Systems, and Relativity, Ed. M.A.H. MacCallum, Cambridge University Press, Cambridge, page 237, 1985.

[18] I. Ciufolini. Generalized geodesic deviation equation. Phys. Rev. D, 34:1014, 1986.

[19] S. L. Bażański and N. N. Kostyukovich. Kinematics of relative motion of charged test particles in general relativity. I. The first electromagnetic deviation. Acta Phys. Pol. B, 18:601, 1987.

[20] S. L. Bażański and N. N. Kostyukovich. Kinematics of relative motion of charged test particles in general relativity. II. The second electromagnetic deviation. Acta Phys. Pol. B, 18:621, 1987.

[21] S. L. Bażański. Hamilton-Jacobi formalism for geodesics and geodesic deviations. J. Math. Phys. (N.Y.), 30:1018, 1989.

[22] L. Vanzo. A generalization of the equation of geodesic deviation. Nuovo Cim. B, 107:771, 1992.

[23] R. Kerner, J. W. van Holten, and R. Colistete Jr. Relativistic epicycles: another approach to geodesic devia- tions. Class. Quantum Grav., 18:4725, 2001.

[24] S. Manoff. Deviation operator and deviation equations over spaces with affine connections and metrics. J. Geom. Phys., 39:337, 2001.

[25] J. W. van Holten. World-line deviations and epicycles. Int. J. Mod. Phys. A, 17:2764, 2002.

[26] C. Chicone and B. Mashhoon. The generalized Jacobi equation. Class. Quant. Grav., 19:4231, 2002.

[27] V. Perlick. On the generalized Jacobi equation. Gen. Rel. Grav, 40:1029, 2008.

[28] J. Vines. Geodesic deviation at higher orders via covariant bitensors. Gen. Rel. Grav, 47:59, 2015.

[29] F. W. Hehl, P. von der Heyde, G. D. Kerlick, and J. M. Nester. General relativity with spin and torsion: Foundations and prospects. Rev. Mod. Phys., 48:393, 1976.

[30] M. Blagojević and F. W. Hehl. Gauge theories of gravitation: A reader with commentaries. Imperial College Press, London, 2013.

[31] V. N. Ponomarev, A. O. Barvinsky, and Y. N. Obukhov. Gauge approach and quantization methods in gravity theory. Nauka, Moscow, 2017.

[32] F. W. Hehl, Yu. N. Obukhov, and D. Puetzfeld. On Poincaré gauge theory of gravity, its equations of motion, and Gravity Probe B. Phys. Lett. A, 377:1775, 2013.

[33] D. Puetzfeld and Yu. N. Obukhov. Equations of motion in metric-affine gravity: a covariant unified framework. Phys. Rev. D, 90:084034, 2014.

[34] Yu. N. Obukhov and D. Puetzfeld. Multipolar test body equations of motion in generalized gravity theories. "Equations of Motion in Relativistic Gravity", D. Puetzfeld et. al. (eds.), Fundamental theories of Physics, Springer, 179:67, 2015.

[35] A. Trautman. Einstein-Cartan theory. Encyclopedia of Mathematical Physics, edited by J.-P. Francoise, G.L. Naber and S.T. Tsou, Oxford: Elsevier, 2:189, 2006.

[36] Yu. N. Obukhov. Poincaré gauge gravity: selected topics. Int. J. Geom. Meth. Mod. Phys., 03:95, 2006.

[37] W. H. Goldthorpe. Spectral geometry and $S O(4)$ gravity in a Riemann-Cartan spacetime. Nucl. Phys. B, 170:307, 1980.

[38] H. T. Nieh and M. L. Yan. Quantized Dirac field in curved Riemann-Cartan background: I. Symmetry properties, Green's function. Annals of Physics (N.Y.), 138: 237, 1982.

[39] N. H. Barth. Heat kernel expansion coefficient: I. An extension. J. Phys. A: Math. Gen., 20:857, 1987.

[40] S. Yajima. Evaluation of the heat kernel in RiemannCartan space. Class. Quant. Grav., 13:2423, 1996.

[41] S. S. Manoff. Auto-parallel equation as Euler-Lagrange's equation in spaces with affine connections and metrics. Gen. Rel. Grav., 32:1559, 2000. URL gr-qc/0010048.

[42] S. S. Manoff. Deviation equations of Synge and Schild over spaces with affine connections and metrics. Int. J. Mod. Phys., A16:1109, 2001. URL gr-qc/0012015.

[43] B. Z. Iliev. Deviation equations in spaces with a transport along paths. JINR communication, E2-94-40, Dubna, 1994, 2003. URL math-ph/0303002.

[44] R. J. van den Hoogen. Towards a covariant smoothing procedure for gravitational theories. J. Math. Phys., 58: 122501, 2017.

[45] J. L. Synge. Relativity: The general theory. North- 
Holland, Amsterdam, 1960.

[46] E. Poisson, A. Pound, and I. Vega. The motion of point particles in curved spacetime. Living Reviews in Relativity, 14(7), 2011.

[47] P. Szekeres. The gravitational compass. J. Math. Phys., 6:1387, 1965.

[48] I. Ciufolini and M. Demianski. How to measure the curvature of space-time. Phys. Rev. D, 34:1018, 1986.

[49] O. Veblen. Normal coordinates for the geometry of paths. Proc. Nat. Acad. Sci. (USA), 8:192, 1922.

[50] O. Veblen and T. Y. Thomas. The geometry of paths. Trans. Amer. Math. Soc., 25:551, 1923.

[51] T. Y. Thomas. The differential invariants of generalized spaces. Cambridge: University Press, 1934.

[52] J. A. Schouten. Ricci-Calculus. An introduction to tensor analysis and its geometric applications. Springer, Berlin, 2nd edition, 1954.

[53] I. G. Avramidi. A covariant technique for the calculation of the one-loop effective action. Nucl. Phys. B, 355:712, 1991.

[54] I. G. Avramidi. Covariant methods for the calculation of the effective action in quantum field theory and investigation of higher-derivative quantum gravity. 1995. URL hep-th/9510140.

[55] A. Z. Petrov. Einstein spaces. Pergamon Press: Oxford, New York, 1969. 